\title{
Identifying Emerging Trends and Temporal Patterns about Self-driving Cars in Scientific Literature
}

\author{
Workneh Y. Ayele ${ }^{1}$ and Imran Akram ${ }^{1}$ \\ ${ }^{1}$ Stockholm University, Department of Computer and Systems Science, Sweden \\ workneh@dsv.su.se, imran.akramegmail.com
}

\begin{abstract}
Self-driving is an emerging technology which has several benefits such as improved quality of life, crash reductions, and fuel efficiency. There are however concerns regarding the utilization of self-driving technology such as affordability, safety, control, and liabilities. There is an increased effort in research centers, academia, and the industry to advance every sphere of science and technology yet it is getting harder to find innovative ideas. However, there is untapped potential to analyze the increasing research results using visual analytics, scientometrics, and machine learning. In this paper, we used scientific literature database, Scopus to collect relevant dataset and applied a visual analytics tool, CiteSpace, to conduct co-citation clustering, term burst detection, time series analysis to identify emerging trends, and analysis of global impacts and collaboration. Also, we applied unsupervised topic modeling, Latent Dirichlet Allocation (LDA) to identify hidden topics for gaining more insight about topics regarding self-driving technology. The results show emerging trends relevant to self-driving technology and global and regional collaboration between countries. Moreover, the result form the LDA shows that standard topic modeling reveals hidden topics without trend information. We believe that the result of this study indicates key technological areas and research domains which are the hot spots of the technology. For the future, we plan to include dynamic topic modeling to identify trends.
\end{abstract}

Keywords: Self-driving Car, Clustering, Term Burst Detection, Time Series Analysis, Visual Analytics.

\section{Introduction}

Self-driving or autonomous driving is an emerging automotive technology [1], and it has been an interesting topic since the 1920s [2]. The evolution of self-driving vehicles has progressed from radio-based to vision and electronic-based systems. Self-driving cars were controlled in the 1920 s by radio, 1960 s by electronic, and 1980 s by vision \& electronic systems [2]. The emergence of self-driving technology may presumably indicate concerns, increasing interests in the use of technology, and market potential. There are increasing concerns such as the impact of self-driving technologies on urban 
planning [3], ethical issues [4], transportation system, affordability, safety, control and liabilities [5]. Despite these concerns, self-driving technologies have several benefits [3].

There are several benefits of utilizing self-driving cars such as promoting safety and improving quality of life [5], efficient parking, optimum driving time, crash reductions and fuel efficiency [6]. Hence, as a disruptive technology, research regarding selfdriving cars and self-driven vehicles is valuable as the technology touches a broader sphere of research domains. Also, stakeholders engaged in research, prototyping and manufacturing benefit by collaboration with universities and research centers. Collaboration between academia and industry, despite being mutually beneficial, is often challenging, not only because of varying pace but also because of the different goals of these stakeholders [7]. In addition to that, there is also a communication gap between academia and the industry [8]. Brijs argues that there is no easy way to bridge the communication gap [8]. However, technology incubators, research centers, and technology transfer agents play an important role in bridging this communication gap [9]. Furthermore, despite the exponential growth of research results from increased research efforts, it is getting harder to find ideas [10].

Trend analysis can be used for forecasting trends in technology [11]. Analyzing and identifying emerging trends can be beneficial for the decision makers and stakeholders in academia and the industry such as financiers, universities, companies and academic publishers [12]. For example, identification of emerging trends of topics in science and technology is essential for making decisions [13]. Therefore, in this study, we aim to identify emerging trends about self-driving cars using academic research findings by applying visual analytics and machine learning.

The research question is "What are emerging trends in auto-industry, in particular, self-driving cars?" To answer this research question, we applied visual analytics on a scientific literature dataset by applying co-citation clustering, time series analysis of clusters, time-series analysis of term burst detection based on [14], Kleinberg's, algorithm. Temporal patterns are measured using citation burstiness [15]. Also, we analyzed the visualization of global collaboration on Google Earth using CiteSpace generated geospatial data. A corpus containing a total of 3323 was collected from Scopus on September 20, 2018. The collected data spans from 1976 to 2019 of which 18 articles were obtained that are accepted for publication in 2019. We chose Scopus because it covers most recent scientific journals [16] and larger databases than Web of Science [17].

Finally, we used the same dataset and run topic identification using Latent Dirichlet Allocation (LDA) and RStudio ${ }^{1}$ and identified 20 hidden topics following the use case presented by [3]. We then compared these 20 topics with trending topics identified by using CiteSpace. The comparison shows that unless temporal, dynamic topic modeling is used, standard topic modeling techniques do not reveal trends of topics. Therefore, for the future, we plan to use dynamic topic modeling to identify temporal trends and compare them with the results of CiteSpace. In this paper, we present six sections. In the second section, we present the A Review of Trend Identification. From the third to the Sixth Sections we present Methodology, Results, Discussion and Future Directions, and Conclusions.

\footnotetext{
${ }^{1}$ https://www.rstudio.com/products/rstudio/download/
} 


\section{A Review of Trend Identification: Visualization Analytics, Scientometric, Text Mining, Clustering of Co-citation Networks}

In this section, a brief review of methods that are applicable to identify emerging trends such as text mining, co-citation analysis, visual analytics, and scientometrics are presented.

\subsection{Visual Analytics}

The first computer visualization of citation network was done by Yemish in 1975 [18]. Visual analytics applied on scientific literature enables to track the emergence of trends and to identify critical evidence in repeatable, flexible, timely, and valuable approach [19]. Keim et al. define visual analytics as a set of techniques that combines automated analysis with interactive visualizations for an adequate understanding, reasoning \& decision making based on the analysis of large and complex datasets [20]. Visual analytics can be applied to analyze human mobility to understand mobility patterns and movement behaviors to support human perception, cognition, and reasoning [21]. Also, it is possible to depict geographical collaboration of authors using visual analytics, see Section 5.3.

\subsection{Scientometrics}

The study of academic literature research performance measurement and evaluation of quantitative aspects of communication of science as a system is referred to as scientometrics [22]. Trend analysis using co-citation and co-word detection for either policy or academic purposes could be used to detect research trends [23]. In addition to visualization functionalities, text mining functionalities are becoming common in scientometric tools such as VOSviewer [24]. Scientometric provides the analysis and identification of hot trends by providing insights and reflection for the past and the future [25].

\subsection{Text Mining}

Text mining is a subfield of computer science that combines techniques of natural language processing, knowledge management, data mining, information retrieval, and, machine learning [26]. Moreover, the techniques and tools used in text mining could be used to analyze social media textual data for commercial and research purposes [27].

Natural Language Processing (NLP) covers any natural language manipulation using computers [28]. NLP can use machine learning for linguistic analysis [29]. Capturing emerging trend is also possible using topic modeling [30].

Topic modeling is an evolving technique in machine learning which enables the identification of hidden topics from collections of textual datasets [31]. Blei et al. introduced LDA, a topic modeling technique that uses probabilistic generative model to generate topics by clustering co-occurring terms [32]. Topic detection methods such as dynamic topic models are also applicable to identify and capture emerging trends [30]. 


\section{$2.4 \quad$ Trend Detection}

Trend detection and analysis is the application of computer science and statistics to predict emerging topics [33]. Trend forecasting is the result of an analysis of data from diverse sources. For example, using interviews trends can be forecasted in early warning systems [34]. Similarly, customer rating or word of mouth can be used to assess the market potential of products [35]. It is also possible to use patent analysis for forecasting, for example, trends in technology [11].

Trend analysis can be done using a wide variety of data sources for various applications. For example, trend analysis on social media data is applied for marketing [36], predicting the future of technology [37], emergency management [38- 39], and monitoring diseases propagation [40]. Also, trend analysis can be applied in emergency management and sensing trending topics [41].

\subsection{Clustering of Co-citation Networks: CiteSpace}

CiteSpace $^{2}$ is a freely available tool developed by Drexel University (U.S.A.) which is used to analyze temporal and structural patterns in scientific literature databases [42]. CiteSpace clusters dataset of literature using co-citation network analysis [42]. Cocitation analysis is the main method to analyze structures of scientific works empirically [43]. CiteSpace provides the use of noun phrases of titles $\mathbf{T}$, keywords $\mathbf{K}$ and abstracts A of articles to label clusters identified as suggested by [44]. The labeling of clusters is inspired by three algorithms such as LSI (Latent Semantic Indexing), LLR (LogLikelihood Ratio) and MI (Mutual Information) [45]. LLR often gives the best results concerning coverage and uniqueness [44]. LSI tend to extract clusters to eliciting implicit semantic relationship across the dataset. However, LLR and MI tend to extract a unique aspect of a cluster [45].

Also, Kleinberg's bust-detection algorithm, which is originally designed to detect bursts of single words [14], is used to detect multiword-term bursts and time series analysis of documents [42]. In CiteSpace, the detection of trending terms is identified using burst detection as proposed by [14] which is based on relative frequency of terms. Finally, the visualization of co-citation makes results easily understandable, as the objective of visualization is the portrayal of meaningful structures [18].

Some of the major metrics used in CiteSpace are centrality measures, burstiness of citations, silhouette and modularity. Centrality measure, ranges between 0 and 1 , measures betweenness score that indicates how different clusters are connected in CiteSpace [42]. Burstiness of citations are surges of citation which enables the identification of developing interests of a research area in academia, the higher the value the higher is the intensity of the burst [42]. Silhouette value ranges from -1 to 1 and shows the homogeneity of a cluster. Higher values of silhouette represent more consistency among cluster members given that the clusters in comparison are similar in size [44]. Modularity measures the extent to which a network can be decomposed into independent blocks or modules. This ranges between 0 and 1 where low modularity suggests that the network cannot be reduced to clusters with clear boundaries, whereas high modularity represents a well-structured network [46].

\footnotetext{
${ }^{2}$ http://cluster.ischool.drexel.edu/ cchen/citespace/download/
} 


\subsection{Related Research}

Marçal et al. used Web of Science datasets and scientometric research approach to identify gaps in prospective studies of self-driving vehicles [47]. Review of trend identification techniques using text mining was done 15 years ago [48]. These trend detection techniques were used in diverse domains of research. Also, there are plenty of academic research findings in the literature that uses visualization analytics, scientometrics, and text mining such as topic modeling to identify trends. However, trend detection research regarding self-driving cars is rare.

\section{$3 \quad$ Methodology}

The data was extracted from Scopus, and CiteSpace was used on the dataset to generate co-citation clustering, visualizations, labeling of clusters, geographical visualization and analysis and interpretation following [42]. We used Kleinberg's burst detection implementation [14] of CiteSpace to identify temporal patterns. A burst detection algorithm determines whether short interval variations of frequency fluctuation as determined by its frequency functions are statistically significant or not during the overall period [15]. Additionally, the $\mathrm{R}^{3}$ statistical tool was used to generate topic modeling to identify hidden topics following [32] and using the use case described in [3].

For identifying trends, it is possible to use tools and algorithms through scientometrics, topic modeling, time-series-analysis of co-citation and co-occurring terms as described in Section 2. We choose CiteSpace because it is freely available and suitable for visualizing trends and patterns. On the other hand, IN-SPIRE could be used for the identification of trends, yet it is commercially available, and other software tools are more focused on other features [49]. Besides, CiteSpace is currently updated frequently, for example, it was updated six times from March to August 2018. Also, topic modeling is applicable to explore trends and themes from a collection of scientific literature [50]. LDA is the most widely used unsupervised technique for topic modeling [51]. In this study, we used standard non-dynamic LDA to complement CiteSpace by identifying topics without considering trends; and to compare trendy topics generated by CiteSpace with non-trendy topics generated by LDA.

\subsection{Computing Tools Used}

A 64-bit operating system, Windows 10 Enterprise, running on an x64-based processor with $2.6 \mathrm{GHz}$ Eight-Core Processor, $20 \mathrm{~GB}$ RAM was used. For merging datasets and processing Notepad and Microsoft Excel were used. The analysis of the dataset was done using CiteSpace. In this study, we also used R to generate topics using standard LDA.

\subsection{Dataset Extraction}

We carefully applied a search query that enabled us to refine our search to the most relevant dataset. Without a carefully formulated query, we would end up having tens of

\footnotetext{
${ }^{3}$ https://www.r-project.org
} 
thousands of irrelevant data, so we used synonym terms in the query. For example, the term self-driving has many forms as illustrated in the query below. According to [44] irrelevant data is imminent no matter how we formulate our query, yet CiteSpace visualizes irrelevant data in isolated clusters in most cases [44].

The dataset was extracted from Scopus on September 20, 2018, using the query illustrated below. A corpus with a total of 3323 was obtained. The collected data spans from 1976 to 2019 of which 18 articles were obtained from accepted and added to Scopus before they are published in 2019.

Query used:

( (car OR automobile) AND (selfdrive OR selfdriven OR selfdriving OR self-drive OR self-driving $O R$ self-driven $O R$ "self drive" $O R$ "self driven" $O R$ "self driving" $O R$ autopilot $O R$ autonomous $O R$ ("autonomous driving") OR "autonomous drive" OR "autonomous driven")) AND (LIMIT-TO (LANGUAGE, "English"))

\section{$4 \quad$ Results}

The clustering of co-citation shows that there are trends indicating most hot areas of research \& development related to self-driving cars, clustering without time-series visualization is illustrated in Fig 1 with nodes of 747 and 1546 connections. We run CiteSpace with a combination of thresholds of Top 60. These means we used top 60 citations per slice, where the number of years per slice is one. The resulting network has a Silhouette value of 0.39 and Modularity of 0.89 . The quality of clustering configuration is measured by silhouette value while modularity measures the soundness of network structure. Here the modularity is sound while the silhouette is a little lower than 0.5 . This is a fair clustering as it is recommended to balance modularity and silhouette values simultaneously [15]. A total of 186 Clusters where identified and 22 most significant clusters are listed as illustrated in Table 1 below. Out of 186, 22 clusters are more relevant as illustrated in the figures, Fig. 2, 3 and 4 below.

Clustering of co-citation and time series analysis was done in two ways. The first clustering was done using node-type references. The clusters are labeled based on titles as illustrated in Section 4.1 to identify research themes and trends based on themes or research titles. The second analysis as illustrated in Section 4.2 was done using nodetype references and abstract terms. The clusters were labeled based on abstract terms to identify relevant terminologies. Finally, analysis of global collaboration, term burst detection, and topic modeling are presented in Sections 4.3, 4.4 and 4.5 respectively.

\subsection{Clustering Node Type Reference: Labeling using Title and Abstract Terms}

The identified clusters were labeled using the Titles of the manuscripts in the collection of documents as illustrated in Fig. 2. and cluster labeling by abstract terms as illustrated in Fig. 3. is presented, and all the three labeling algorithms presented in Section 2.5 are used. 


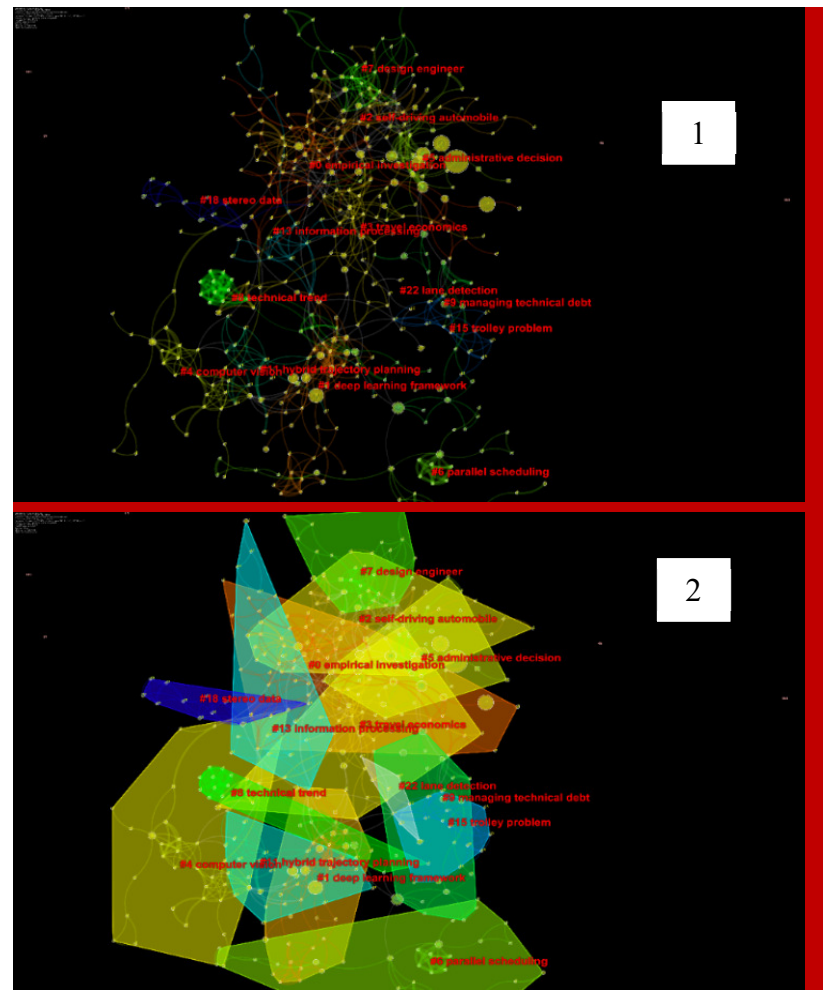

Fig. 1. Co-citation network is colored and labeled based on years, and coloring shows the age of clusters where blue is oldest and greenish and yellowish colors represent newest (1), also (2) each cluster is depicted by a rounded hull colored.

The purpose of analyzing Clusters by title is to identify themes that are commonly discussed among academia from 1976 to 2019. The clusters labeled by title indicates that the most common research themes are an empirical investigation, deep learning framework, travel economics, and computer vision, see Fig 2 below as. 


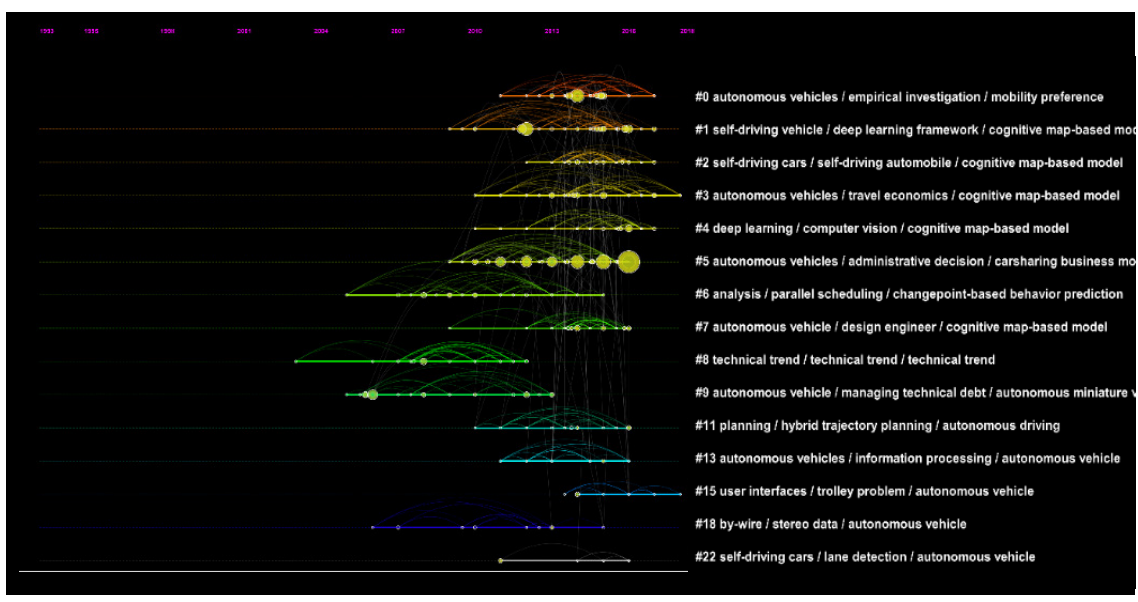

Fig. 2. A timeline analysis of clusters using Titles as cluster labels and node type Reference.

The purpose of analyzing clusters by terms, see Fig. 3, is to identify terms that are representatives of the abstracts between 1976 and 2019. The clusters labeled by terms indicate that the most common research terms are new trip, car detection, transportation network companies, real-world road network, and adversarial attack are the top terms. It is also visible that government agencies are increasingly becoming active participants in self-driving technologies. Also, trolley problem and real-world road networks are currently trending.

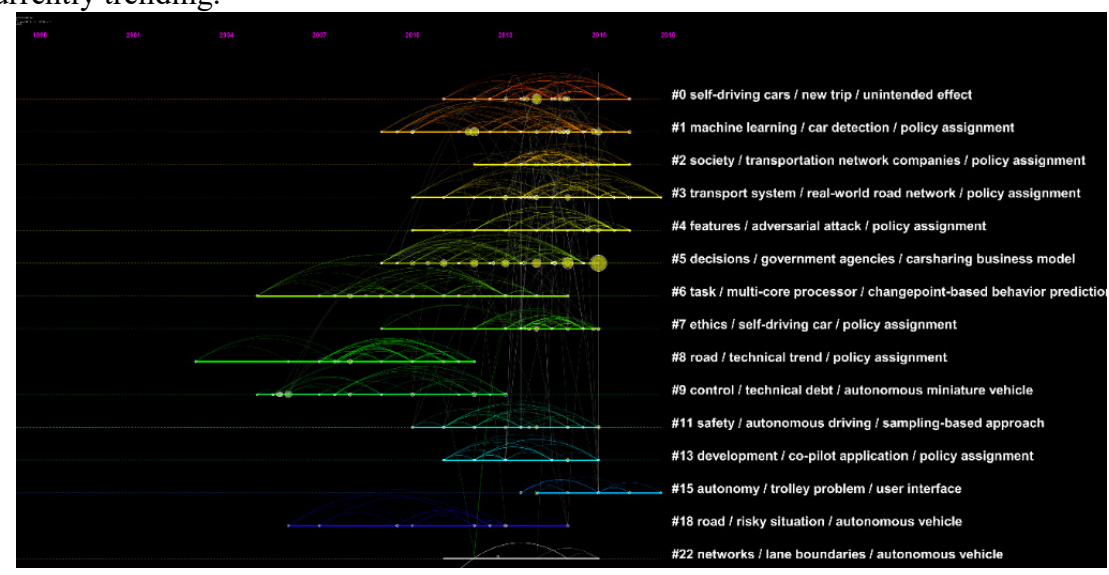

Fig. 3. A timeline analysis of clusters using terms as cluster labels and node type Reference. 


\subsection{Clustering with Node Type Reference and Abstract: Labeling Using Abstract Terms}

In this Section, we present a visualization of a network of clusters generated using node type Reference and Abstract see Fig 4 below. The result shows that there is only one difference on the label of the $11^{\text {th }}$ cluster. When cluster label Abstract is selected, it becomes "collision checking accuracy" instead of "autonomous driving." The term "collision checking accuracy" is more interesting since "autonomous driving" is part of the search query.

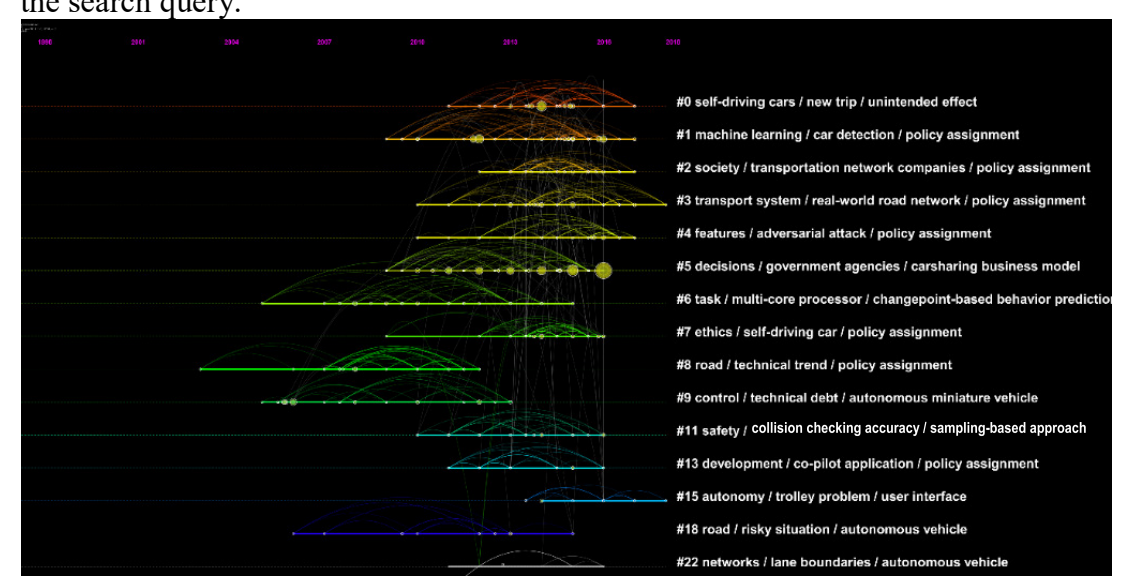

Fig. 4. A timeline analysis of clusters using terms as cluster labels and node type Reference and Term.

To automatically label clusters CiteSpace provides three labeling algorithms such as LSI, LLR, and MI [45] as discussed in Section 2.5. Summary of clusters is presented in Table 1 below. The summary of clusters shows that machine learning, computer vision such as car detection, transport network companies, real-world road network, safety, control, ethics, and other important terms. In this study, LSI and LLR cluster labeling give better and interpretable labeling than MI, see Table 1. The silhouette values of most clusters are greater than 0.5 and are closer to 1 which shows that clusters have more consistency as suggested in [44]. The Mean Year indicates most of the biggest clusters are within the range of five years period and hence they are recent.

Table 1. Summary of term clusters sorted by size and labeling using clustering algorithms LSI, LLR, and MI.

\begin{tabular}{|c|c|c|c|c|c|c|}
\hline$\stackrel{\stackrel{\vec{\omega}}{\Xi}}{\Xi} \theta$ & Size & Silhouette & $\begin{array}{l}\text { Mean } \\
\text { (Year) }\end{array}$ & $\begin{array}{l}\text { Label } \\
\text { (LSI) }\end{array}$ & $\begin{array}{l}\text { Label } \\
\text { (LLR) }\end{array}$ & Label (MI) \\
\hline 0 & 45 & 0.775 & 2014 & $\begin{array}{l}\text { self-driving } \\
\text { cars }\end{array}$ & new trip & user preference \\
\hline 1 & 42 & 0.875 & 2014 & $\begin{array}{l}\text { machine } \\
\text { learning }\end{array}$ & car detection & policy assignment \\
\hline
\end{tabular}




\begin{tabular}{|c|c|c|c|c|c|c|}
\hline 2 & 41 & 0.854 & 2015 & society & $\begin{array}{l}\text { transportation } \\
\text { network companies }\end{array}$ & policy assignment \\
\hline 3 & 33 & 0.831 & 2014 & $\begin{array}{l}\text { transport } \\
\text { system }\end{array}$ & $\begin{array}{l}\text { real-world road } \\
\text { network }\end{array}$ & policy assignment \\
\hline 4 & 31 & 0.902 & 2015 & features & adversarial attack & policy assignment \\
\hline 5 & 31 & 0.875 & 2013 & decisions & $\begin{array}{l}\text { government } \\
\text { agencies }\end{array}$ & allowing car use \\
\hline 6 & 29 & 0.992 & 2011 & task & $\begin{array}{l}\text { multi-core } \\
\text { processor }\end{array}$ & policy assignment \\
\hline 7 & 27 & 0.901 & 2014 & ethics & self-driving car & policy assignment \\
\hline 8 & 19 & 0.981 & 2008 & road & autonomous driving & policy assignment \\
\hline 9 & 19 & 0.947 & 2008 & control & technical debt & actor layer \\
\hline 11 & 18 & 0.943 & 2013 & safety & $\begin{array}{l}\text { collision checking } \\
\text { accuracy }\end{array}$ & $\begin{array}{l}\text { sampling-based } \\
\text { approach }\end{array}$ \\
\hline 13 & 16 & 0.928 & 2013 & development & co-pilot application & policy assignment \\
\hline 15 & 14 & 0.978 & 2015 & autonomy & trolley problem & current finding \\
\hline 18 & 11 & 0.96 & 2011 & road & risky situation & policy assignment \\
\hline 22 & 7 & 0.989 & 2014 & networks & lane boundaries & autonomous vehicle \\
\hline
\end{tabular}

\subsection{Analysis of Global Collaboration: Google Earth Visualization}

Google earth application is used along with a file generated from CiteSpace to explore authors' geographical location and associations to their collaborators as illustrated below. The red links are recent links, and the green ones are old links. As shown in Fig 5 the general development in self-driving cars has become an emerging technological phenomenon since most connections are reddish.

The U.S.A. and Brazil are active in self-driving cars research. With the U.S.A. being the most active country in undertaking and collaboration with other continentals in the region. Europe has more geographically concentrated self-driving car research and collaboration than any other continent. EU member states collaborate within the EU and at the continental level. Countries such as Germany, the U.K., Ireland, France, Sweden, Netherlands, Denmark, Austria, Switzerland, Spain, Italy, Romania, and Poland are among the most active European countries. Japan, South Korea, Taiwan, and China are also actively working on self-driving technology. However, Africa, South America except for Brazil, and the Middle East, in general, are inactive in self- 
driving

technology.

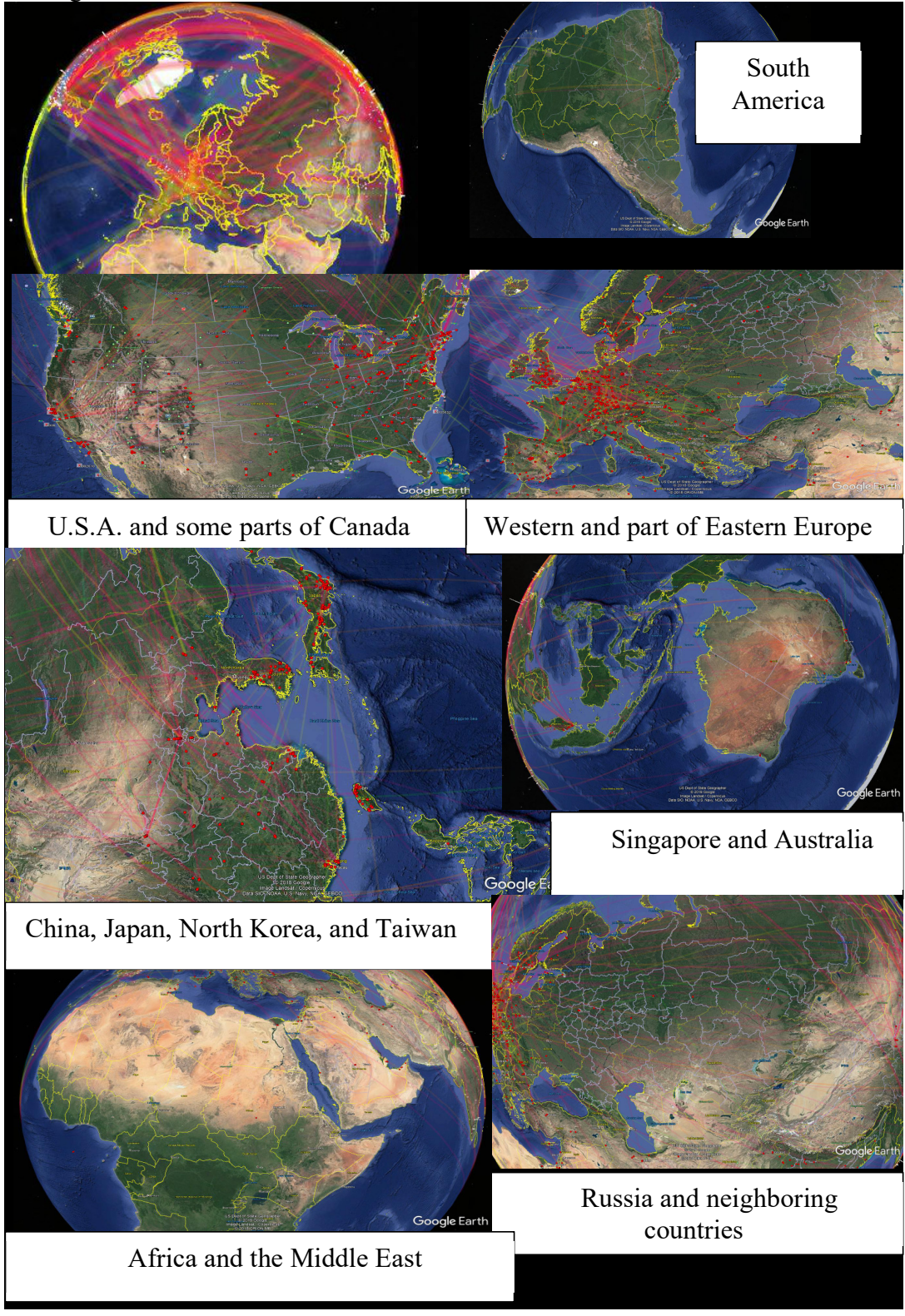

Fig. 5. Google Earth Worldwide Visualization of Research Collaboration on the Topic of Selfdriving Cars. 


\subsection{Term Burst Detection}

We used the default configuration of burst detection function values of CiteSpace. Additionally, we selected Node Types Term and Keyword to identify busty terms and their variation. We selected the top 65 terms per a year slice with Top $\mathrm{N} \%=100 \%$ and G-Index $=7$. This resulted in 87 burst terms as illustrated below in Fig 6 . The burst terms indicate technological terms and their trends through the years 1976 to 2019. Hence it shows which terms are currently active in academia, see Fig. 6. For a full list of termbursts refer to Appendix 1. The result shows that deep learning, convolutional neural network, object detection, neural network, learning system, Internet of Things (IoT), deep neural network, automobile safety device, behavioral research, digital storage, network security, semantics, advanced driver assistance, decision making, traffic sign, image segmentation, and human-computer interaction are currently trending terms.

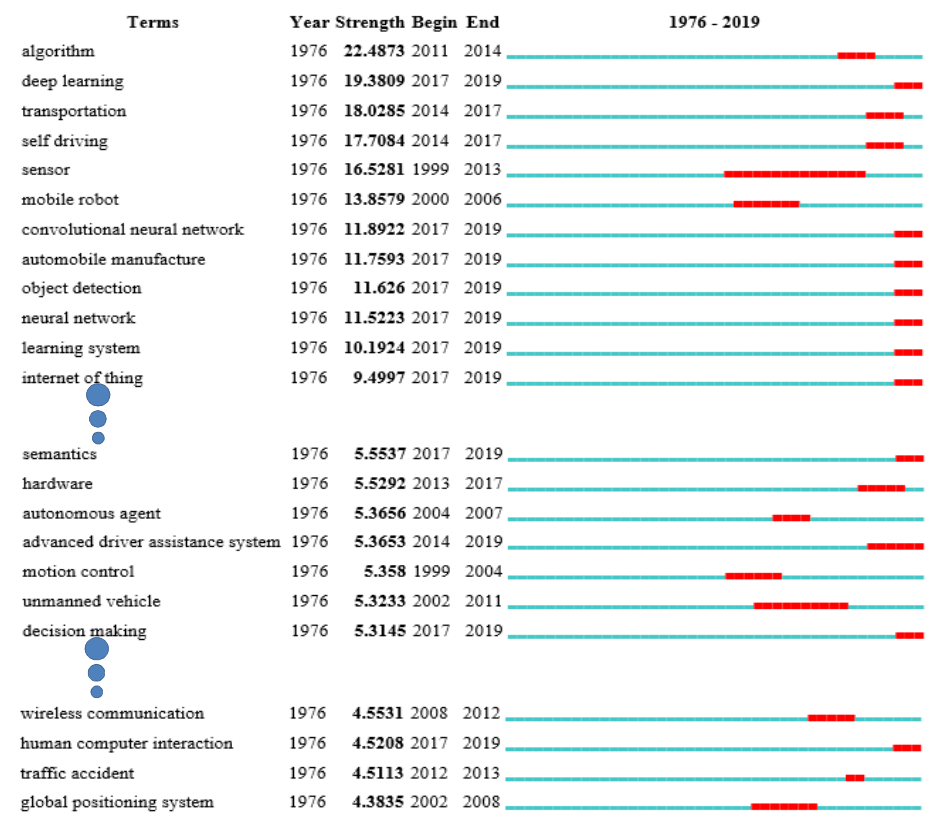

Fig. 6. Term and keyword burst detection and trends from 1976 to 2019. 


\subsection{Topic Modeling}

Topic modeling to identify hidden topic was run using R, and we identified 20 topics. After obtaining the dataset text pre-processing such as removing stopwords, stemming \& stem completion, remove special characters, removing numbers \& punctuations, change cases to lower case, visualization of frequency to check the validity of frequent terms, and conversion of major vocabulary into US English from British English for consistency was done. After preprocessing, we chose the optimum topic number to be generated as described in [3]. The LDA topic model was validated applying a hold out of 10 -fold cross validation using $75 \%$ training-set and $25 \%$ test-set using candidate number of topics, and the evaluation shows that the generated LDA model is suitable if greater than 20 topics are chosen. Some of the major topics identified are safety, car human interaction, computer vision \& pattern recognition, urban planning to accommodate self-driving cars, navigation systems, and machine learning. However, these topics do not reflect any trend as time is not considered in standard topic modeling algorithms. Hence, these generated topics are all identified by CiteSpace in trending and non-trending topics.

\section{$5 \quad$ Discussion and Future Directions}

The purpose of this study is to identify emerging trends in self-driving cars. The result of this study enabled the visualization of existing trends regarding self-driving cars. Recent academic findings indicate that there are concerns related to using self-driving vehicles - for example, control and security [52], land management policy and marketing [53]. The result of the study also suggests that there are concerns related to safety, control, security, urban traffic management, and others.

In this study, CiteSpace is used because it has more visualization options than most exiting visualization tools reviewed. The limitation of this study is that due to time limitation the results are not compared with other visualization tools. For the future, we plan to compare other visualization tools and make recommendations regarding the utilization of existing tools. Also, since LDA only shows hidden topics covered in the discourse of the scientific literature disregarding temporal patterns we plan to apply dynamic topic modeling techniques to identify trending topics and compare them with visual analytics and scientometric tools.

\section{Conclusions}

Knowledge processing through the utilization of the growing academic research findings and patents serves many purposes. There is an exponential growth of research findings, and it has become difficult to find innovative ideas [10]. The problem with finding innovative ideas can be a result of lack of communication between academia and the industry. For example, Brijs argue that there is a communication gap between the academic sector and the industry and there is no easy way to bridge this gap [8]. To circumvent the communication gap which hinders the implementation and commercialization of new technology it is possible to analyze the growing academic research findings to identify emerging trends. This means the industry could utilize the results of the academic realm all over the world in a systematic way through generated insights and trends. 
Identification of new technological advancement enables the elicitation of valuable ideas. Technology incubators, research centers, and technology transfer agents are dedicated to bridging the communication barriers hindering industry-academia collaboration [9]. Therefore, the identification of emerging trends illustrated in this paper can serve technology incubators, research centers, and technology transfer agents.

From the results of this study we arrive at the following conclusions, the most important and trending areas of research are:

1. Machine learning

a. Neural network - deep neural network and convolutional neural network

2. Computer vision, robotics and IoT

a. Sensor

b. Object detection

c. Image segmentation

d. Network security

e. Human-computer interaction

f. Control and safety

3. Social and human aspects

a. Trolley problems, ethics, and behavioral research

b. Safety and security

4. Urban planning and policy

a. Transport network companies

b. Traffic line management

c. Government agencies

Finally, from the analysis of global collaboration, we can deduce that African, the Middle East, South American, and some Asian countries are behind the rest of the world. Also, most European countries collaborate on self-driving technology with other EU member states and with other countries mostly with the United States. Some Asian countries such as Japan, South Korea, Taiwan, China, and Singapore are also active in self-driving research. Also, potential regions where self-driving cars could be tested, and their applicability could be investigated could be African countries, the Middle East, and most Latin America countries. Brazil, however, is more active than most South American countries.

\section{References}

1. Davidson P., Spinoulas A.: Autonomous vehicles: what could this mean for the future of transport, In Australian Institute of Traffic Planning and Management (AITPM) National Conference, Brisbane, Queensland, (2015).

2. Bimbraw, K.: Autonomous cars: Past, present and future a review of the developments in the last century, the present scenario and the expected future of autonomous vehicle technology, In Informatics in Control, Automation and Robotics, ICINCO, 12th International Conference, vol 1. IEEE, pp. 191-198 (2015). 
3. Ayele, W. Y., Juell-Skielse, G.: Unveiling Topics from Scientific Literature on the Subject of Self-driving Cars using Latent Dirichlet Allocation. In 2018 IEEE 9th Annual Information Technology, Electronics and Mobile Communication Conference, IEMCON, pp. 1113-1119. IEEE (2018).

4. Lin, P.: Why ethics matters for autonomous cars. In Autonomous driving, pp. 69-85. Springer, Heidelberg (2016).

5. Howard D., Dai D., Public perceptions of self-driving cars: The case of Berkeley, California, In Transportation Research Board 93rd Annual Meeting Vol. 14(4502), (2014).

6. Fagnant D. J., Kockelman K., "Preparing a nation for autonomous vehicles: opportunities, barriers and policy recommendations", Transportation Research Part A: Policy and Practice, 77, pp. 167-181 (2015).

7. Sandberg, A. B., Crnkovic, I.: Meeting Industry-Academia Research Collaboration Challenges with Agile Methodologies. In Software Engineering: Software Engineering in Practice Track, ICSE-SEIP IEEE/ACM 39th International Conference, IEEE, pp. 73-82 (2017).

8. Brijs, K.: Collaboration between Academia and Industry: KU Leuven. Cereal Foods World, 62(6), 264-266 (2017).

9. Villani, E., Rasmussen, E., Grimaldi, R.: How intermediary organizations facilitate university-industry technology transfer: A proximity approach. Technological Forecasting and Social Change, vol. 114, pp. 86-102 (2017).

10. Bloom, N., Jones, C. I., Van Reenen, J., Webb, M.: Are ideas getting harder to find? (No. w23782). National Bureau of Economic Research (2017).

11. You, H., Li, M., Hipel, K. W., Jiang, J., Ge, B., Duan, H.: Development trend forecasting for coherent light generator technology based on patent citation network analysis. Scientometrics. 111, 1, 297-315 (2017).

12. Salatino, A., Osborne, F., Motta, E.: AUGUR: Forecasting the Emergence of New Research Topics. In JCDL'18: The 18th ACM/IEEE Joint Conference on Digital Libraries. ACM, New York, NY, USA (2018).

13. Small, H., Boyack, K. W., Klavans, R.: Identifying emerging topics in science and technology. Research Policy, 43(8), pp. 1450-1467 (2014).

14. Kleinberg, J.: Bursty and hierarchical structure in streams. Data Mining and Knowledge Discovery, 7(4), 373-397 (2003).

15. Chen, C., Ibekwe-SanJuan, F., Hou, J.: The structure and dynamics of cocitation clusters: A multiple-perspective cocitation analysis. Journal of the American Society for Information Science and Technology, 61(7), pp. 1386-1409 (2010).

16. Aghaei C.A., Salehi H., Yunus M., Farhadi H., Fooladi M., Farhadi M., Ale E.N., A comparison between two main academic literature collections: Web of Science and Scopus databases, (2013).

17. Mongeon P., Paul-Hus A.: "The journal coverage of Web of Science and Scopus: a comparative analysis", Scientometrics, 106(1), pp. 213-228 (2016).

18. Small, H.: Visualizing science by citation mapping. Journal of the American society for Information Science, 50(9), pp. 799-813 (1999).

19. Chen, C., Hu, Z., Liu, S., Tseng, H.: Emerging trends in regenerative medicine: a scientometric analysis in CiteSpace. Expert opinion on biological therapy 12(5), pp. 593608 (2012).

20. Keim, D., Andrienko, G., Fekete, J. D., Görg, C., Kohlhammer, J., Melançon, G.. Visual Analytics: Definition, process, and challenges. In Information Visualization (pp. 154-175). Springer, Berlin, Heidelberg (2008). 
21. Andrienko, G., Andrienko, N., Wrobel, S. Visual analytics tools for analysis of movement data. ACM SIGKDD Explorations Newsletter, 9(2), 38-46 (2007).

22. Mingers, J., Leydesdorff, L.: A review of theory and practice in scientometrics. European Journal of Operational Research, 246(1), pp. 1-19 (2015).

23. Zitt, M., Bassecoulard, E.: Development of a method for detection and trend analysis of research fronts built by lexical or cocitation analysis. Scientometrics, 30, 333-351 (1994).

24. Van Eck, N. J., Waltman, L.: Text mining and visualization using VOSviewer. arXiv preprint arXiv:1109.2058 (2011).

25. Tseng, Y. H., Lin, Y. I., Lee, Y. Y., Hung, W. C., Lee, C. H.: A comparison of methods for detecting hot topics. Scientometrics, 81(1), pp. 73-90 (2009).

26. Feldman, R., Sanger, J.: The text mining handbook: advanced approaches in analyzing unstructured data. Cambridge university press (2007).

27. Hu, X., Liu, H.: Text analytics in social media. Mining text data, pp 385-414 (2012).

28. Bird, S., Klein, E., Loper, E.: Natural language processing with Python: analyzing text with the natural language toolkit. O'Reilly Media, Inc (2009)

29. Sidorov, G., Velasquez, F., Stamatatos, E., Gelbukh, A., Chanona-Hernández, L.: Syntactic n-grams as machine learning features for natural language processing. Expert Systems with Applications 41(3), pp. 853-860 (2014).

30. AlSumait, L., Barbará, D., Domeniconi, C.: On-line lda: Adaptive topic models for mining text streams with applications to topic detection and tracking. In Data Mining, 2008. ICDM'08. Eighth IEEE International Conference, pp. 3-12. IEEE (2008).

31. Blei, D.M.: Probabilistic topic models. Communications of the ACM, 55(4), , pp. 77-84 (2012).

32. Blei, D.M., Ng, A.Y., Jordan, M.I.: Latent Dirichlet Allocation. Journal of machine learning research, pp. 993-1022 (2003).

33. Kataria, D.: A Review on Social Media Analytics. International Journal of Advance Research, Ideas and Innovations in Technology, 3(2), pp. 695-698 (2017).

34. Hando, J., Darke, S., O'brien, S., Maher, L., Hall, W.: The development of an early warning system to detect trends in illicit drug use in Australia: the Illicit Drug Reporting System. Addiction Research, 6(2), 97-113 (1998).

35. Moe, W. W., Trusov, M.: The value of social dynamics in online product ratings forums. Journal of Marketing Research, 48(3), 444-456 (2011).

36. He, W., Zha, S., Li, L.: Social media competitive analysis and text mining: A case study in the pizza industry. International Journal of Information Management. 33(3), pp. 464$472(2013)$.

37. Asur, S., Huberman, B. A.: Predicting the future with social media. In Web Intelligence and Intelligent Agent Technology (WI-IAT), IEEE/WIC/ACM International Conference, IEEE, 1, pp. 492-499 (2010).

38-Yin, J., Karimi, S., Lampert, A., Cameron, M., Robinson, B., Power, R.: Using social media to enhance emergency situation awareness. In Proceedings of the 24th International Conference on Artificial Intelligence, AAAI Press, pp. 4234-4238 (2015).

39. Pohl, D., Bouchachia, A., Hellwagner, H.: Automatic sub-event detection in emergency management using social media. In Proceedings of the 21 st International Conference on World Wide Web, ACM, 683-686 (2012).

40. Corley, C., Mikler, A. R., Singh, K. P., Cook, D. J.: Monitoring Influenza Trends through Mining Social Media, In BIOCOMP, pp. 340-346 (2009).

41. Ayele, W. Y., Juell-Skielse, G.: Social media analytics and internet of things: survey. In Proceedings of the 1st International Conference on Internet of Things and Machine Learning, ACM, p. 53. (2017). 
42. Chen, C.: CiteSpace II: Detecting and visualizing emerging trends and transient patterns in scientific literature. Journal of the American Society for Information Science and Technology, 57(3), 359-377 (2006).

43. Gmür, M.: Co-citation analysis and the search for invisible colleges: A methodological evaluation. Scientometrics, 57(1), pp. 27-57 (2003).

44. Chen, C.: The CiteSpace Manual (Version 0.65). http.//cluster.ischool.drexel.edu/cchen/citespace/CitespaceManual.pdf. 2014-04-06 (2014).

45. Zhu, Y., Kim, M. C., Chen, C.: An investigation of the intellectual structure of opinion mining research. Information Research: An International Electronic Journal, 22(1), n1 (2017).

46. Chen, C, Chen, Y., Horowitz, M., Hou, H, Liu, Z., Pellegrino, D.: Towards an explanatory and computational theory of scientific discovery. Journal of Informetrics, 3(3), 191-209 (2009).

47. Marçal, R., Antonialli, F., Habib, B., Neto, A. D. M., de Lima, D. A., Yutaka, J., ... \& Nicolaï, I.: AUTONOMOUS VEHICLES: Scientometric and bibliometric studies. In 25th International Colloquium of Gerpisa-R/Evolutions. New technologies and services in the automotive industry (2017).

48. Kontostathis, A., Galitsky, L. M., Pottenger, W. M., Roy, S., Phelps, D. J.: A survey of emerging trend detection in textual data mining. In Survey of text mining (pp. 185-224). Springer, New York, NY (2004).

49. Cobo, M. J., López-Herrera, A. G., Herrera-Viedma, E., Herrera, F.: (2011). Science mapping software tools: Review, analysis, and cooperative study among tools. Journal of the American Society for Information Science and Technology 62(7), 1382-1402.

50. Youssef, A., Rich, A.: Exploring trends and themes in bioinformatics literature using topic modeling and temporal analysis. In Systems, Applications and Technology Conference (LISAT), 2018, pp. 1-6. IEEE, Long Island (2018).

51. Gerlach, M., Peixoto, T. P., Altmann, E. G.: A network approach to topic models. Science advances $4(7)$, (2018).

52. Schoettle B., Sivak M., A survey of public opinion about autonomous and self-driving vehicles in the US, the UK, and Australia, (2014).

53. Bansal, K.M. Kockelman P., Are we ready to embrace connected and self-driving vehicles? A case study of Texans, Transportation, 45(2, pp.641-675 (2018). 
Appendix 1: Top 87 Terms with the Strongest Citation Bursts

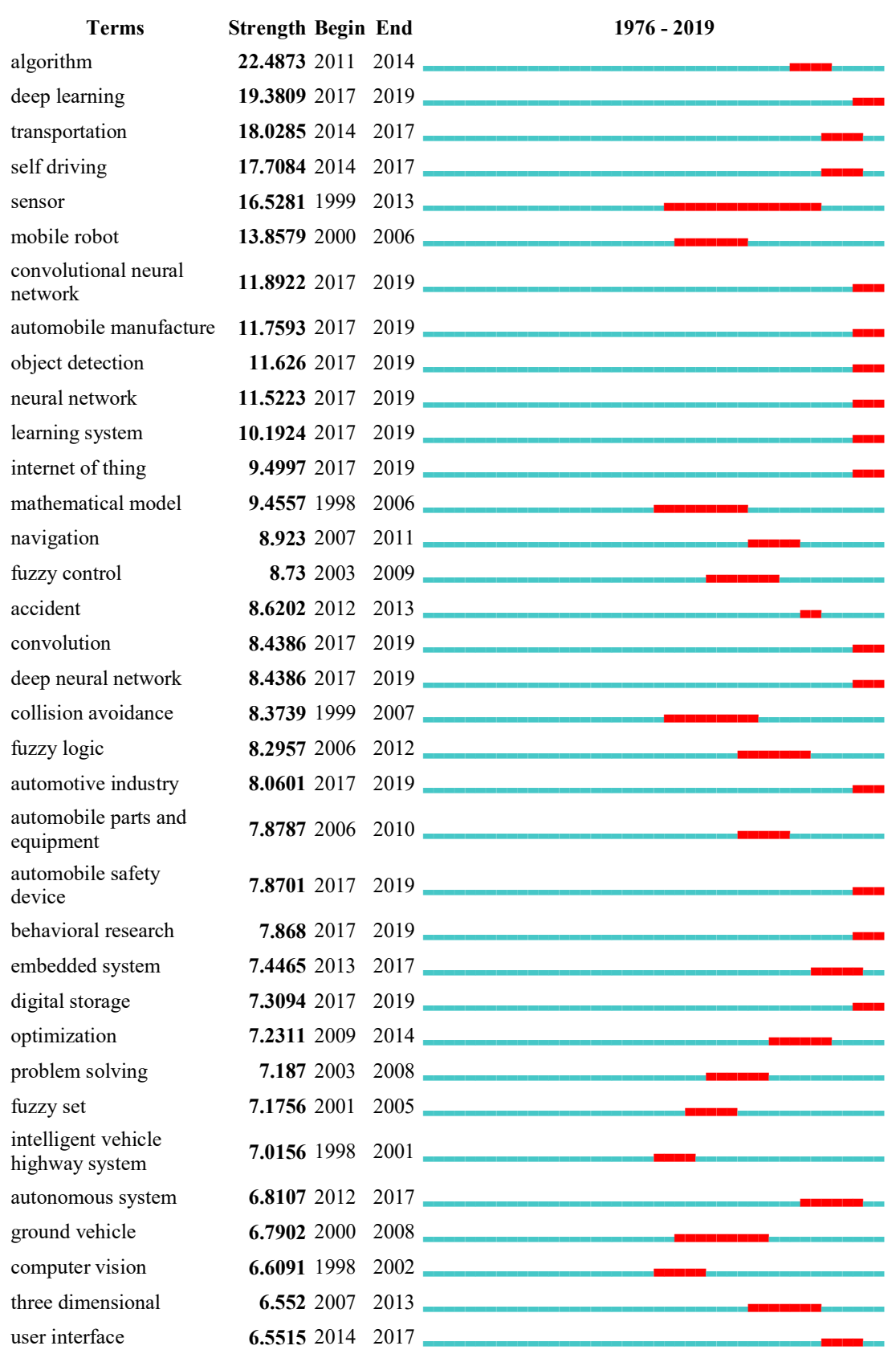




\begin{tabular}{|c|c|c|c|}
\hline control system & 6.4582003 & 2007 & , \\
\hline intelligent robot & 6.34782010 & 2011 & 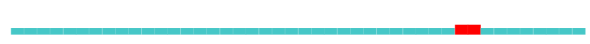 \\
\hline autonomous navigation & 6.29442006 & 2014 & \\
\hline steering & 6.22612002 & 2012 & \\
\hline $\begin{array}{l}\text { cyber physical systems } \\
\text { (cpss) }\end{array}$ & 6.22612013 & 2014 & 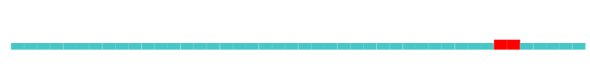 \\
\hline parking & 6.16132004 & 2012 & \\
\hline network security & 6.0563 2017 & 2019 & \\
\hline $\begin{array}{l}\text { automobile electronic } \\
\text { equipment }\end{array}$ & 6.0549 2004 & 2010 & \\
\hline multi agent system & 6.02962005 & 2013 & \\
\hline human & 5.96642012 & 2017 & \\
\hline design & $\mathbf{5 . 8 7 1 3 2 0 0 9}$ & 2014 & \\
\hline computer software & $\mathbf{5 . 8 1 5 8} 2002$ & 2009 & \\
\hline computer simulation & $\mathbf{5 . 7 2 4 2} 2010$ & 2013 & \\
\hline car & 5.66772014 & 2017 & \\
\hline semantics & $\mathbf{5 . 5 5 3 7} 2017$ & 2019 & \\
\hline hardware & $\mathbf{5 . 5 2 9 2} 2013$ & 2017 & \\
\hline autonomous agent & $\mathbf{5 . 3 6 5 6 2 0 0 4}$ & 2007 & \\
\hline $\begin{array}{l}\text { advanced driver } \\
\text { assistance system }\end{array}$ & $\mathbf{5 . 3 6 5 3 2 0 1 4}$ & 2019 & \\
\hline motion control & $\mathbf{5 . 3 5 8} 1999$ & 2004 & \\
\hline unmanned vehicle & $\mathbf{5 . 3 2 3 3} 2002$ & 2011 & \\
\hline decision making & $\mathbf{5 . 3 1 4 5} 2017$ & 2019 & \\
\hline intelligent system & 5.29662012 & 2014 & \\
\hline real time system & 5.28992002 & 2005 & \\
\hline machine design & $\mathbf{5 . 2 4 5 8} 2004$ & 2010 & \\
\hline traffic sign & $\mathbf{5 . 1 9 2 8} 2017$ & 2019 & \\
\hline real time & 5.12192008 & 2011 & \\
\hline intelligent vehicle & $\mathbf{5 . 0 5 9 3 2 0 0 7}$ & 2010 & \\
\hline research & $\mathbf{5 . 0 5 3 6} 2009$ & 2013 & \\
\hline urban environment & $\mathbf{5 . 0 0 7 4} 2010$ & 2014 & \\
\hline image segmentation & 4.92862017 & 2019 & \\
\hline car following & 4.75022010 & 2012 & \\
\hline navigation system & 4.69732006 & 2013 & \\
\hline car like mobile robot & 4.65832003 & 2010 & \\
\hline article & $\mathbf{4 . 5 8 4 2} 2012$ & 2013 & $=$ \\
\hline wireless communication & $\mathbf{4 . 5 5 3 1 2 0 0 8}$ & 2012 & \\
\hline $\begin{array}{l}\text { human computer } \\
\text { interaction }\end{array}$ & 4.52082017 & 2019 & \\
\hline raffic accident & 4.51132012 & 2013 & \\
\hline
\end{tabular}




\begin{tabular}{|c|c|c|}
\hline $\begin{array}{l}\text { global positioning } \\
\text { system }\end{array}$ & $\mathbf{4 . 3 8 3 5} 2002$ & 2008 \\
\hline car like robot & 4.3042012 & 2013 \\
\hline model & 4.27632010 & 2013 \\
\hline accident prevention & 4.23552001 & 2006 \\
\hline brake & 4.1882011 & 2012 \\
\hline electric vehicle & 4.17412011 & 2013 \\
\hline lane detection & 4.12782012 & 2013 \\
\hline robotics & 4.1252000 & 2007 \\
\hline signal processing & 4.11232012 & 2014 \\
\hline safety & 4.08552014 & 2017 \\
\hline fuzzy system & 4.07552008 & 2010 \\
\hline maneuverability & 4.00042003 & 2006 \\
\hline pedestrian safety & 3.92062012 & 2013 \\
\hline intelligent control & 3.912007 & 2013 \\
\hline reinforcement learning & $\mathbf{3 . 8 9 2 5} 2011$ & 2013 \\
\hline
\end{tabular}

